\title{
Generative capacity of sticker systems with the presence of weights
}

\author{
Y.S. Gan ${ }^{\mathrm{a}, *}$, W.H. Fong ${ }^{\mathrm{b}}$, N.H. Sarmin ${ }^{\mathrm{b}}$, S. Turaev ${ }^{\mathrm{c}}$ \\ a Xiamen University Malaysia, Jalan Sunsuria, Bandar Sunsuria, 43900 Sepang, Selangor, Malaysia \\ b Department of Mathematical Sciences, Faculty of Science, Universiti Teknologi Malaysia, \\ 81310 UTM Johor Bahru, Johor, Malaysia \\ c Department of Computer Science, Faculty of Information and Communication Technology, \\ International Islamic University of Malaysia, 53100 Kuala Lumpur, Malaysia
}

*Corresponding author, e-mail: ysgan@xmu.edu.my

Received 22 Aug 2014

Accepted 21 May 2017

\begin{abstract}
DNA computing involves computing models which use the recombination behaviour of DNA molecules as computation devices. This idea was successfully applied by Adleman in his biological experiment in order to show the solvability of the Hamiltonian path problem for larger instances. A DNA-based computation model called a sticker system is an abstraction of the computations using the recombination behaviour as in Adleman's experiment. In this paper, the generative capacity of several variants of bounded delay and unrestricted weighted sticker systems is investigated. The relation between families of languages generated by several variants of weighted sticker systems and weighted grammars is also presented.
\end{abstract}

KEYWORDS: DNA computing, formal language

MSC2010: 68Q15 68Q70 97P20 97M60 93A30

\section{INTRODUCTION}

Computers have been informally defined as electronic devices which are able to receive, manipulate and store the input and produce an output. With the modern technological skills, many supercomputers with microprocessors made from silicon have been produced to match the higher speed of data processing. However, these silicon-based supercomputers are limited in terms of speed and density, design complexity, non-recurring, high cost, power consumption and heat dissipation as stated by Hassan et $\mathrm{al}^{1}$. Hence this motivates new technologies in computational devices to overcome the limitations of the conventional silicon chip supercomputers. One of the new technologies from the intersection of computer sciences and bio-molecular sciences is DNA computing. To overcome the limitation of silicon-based computers, a bio-computer has been proposed at the theoretical level to replace the digital switching primitives to DNA molecules.

There are two features of interest in DNA computing, which are Watson-Crick complementarity of DNA molecules that naturally occurs in DNA molecules, and massive parallelism. Watson-Crick complementarity enables the DNA molecules to use the information encoded on a single-strand to make far-reaching conclusions since the information on the other strand can be decoded according to the complementarity. Massive parallelism of DNA strands allows the construction of many copies of DNA strands to be carried out in parallel. From there, many applications show that these two features of DNA molecules on DNA-based computers can solve many computationally intractable problems, for instance the Hamiltonian path problem ${ }^{2}$, the satisfiability problem for arbitrary contact networks ${ }^{3}$ and the satisfiability problem for Boolean circuits ${ }^{4}$.

In DNA computing, the sticker system, a DNAbased computing device, has been introduced by Kari in $1998^{5}$. The sticker systems are developed by using the sticker operations based on the Watson-Crick complementarity of DNA molecules. The computation process on a sticker system using sticker operation is achieved when complete double-stranded DNA molecules are formed from incomplete DNA dominoes. The incomplete DNA dominoes stick to other dominoes by ligation and annealing. Hence in this paper, we investigate the 
level of generative power among these new variants of weighted sticker systems. We should mention that the ideas of using weights in increasing the generative power of generative devices have been widely investigated in formal language theory ${ }^{6-11}$. Besides, the use of weights in sticker systems helps to construct more accurate models for stochastic phenomena and processes in many applications of sticker systems and formal language theory.

\section{PRELIMINARIES}

In this section, some basic terminology, notation, and definitions in formal language theory, sticker systems, and weighted sticker systems are included. For further information regarding the basic concepts of formal language theory, automata and sticker systems, the reader may refer to Refs. 12-15.

In this paper, we use the general notation as follows. Symbols + and $\times$ denote the usual addition and multiplication operations, respectively. Symbols $\oplus$ and $\otimes$ denote the componentwise addition and multiplication operations, respectively. The sets of integers and positive rational numbers are denoted by $\mathbb{Z}$ and $\mathbb{Q}^{+}$, respectively. The symbol $\mathbb{Z}^{n}$ denotes the $n$-dimensional vector space over integers. The set of matrices with integer entities is denoted by $\mathbb{M}$. The cardinality of a set $X$ is denoted by $|X|$. The complete double-stranded sequence of alphabet $V$ is denoted by $W K_{\rho}(V)$.

In the representation of DNA sequences, the form of

$$
\left[\begin{array}{l}
x \\
y
\end{array}\right]
$$

represents a DNA molecule where $x$ and $y$ are the upper DNA strand and lower DNA strand, respectively, with a precise bonding between the corresponding symbols in the strands $x$ and $y$. Furthermore, for some symbols $x, y$, and $W K_{\rho}(V)$, we shall write

$$
\left[\begin{array}{l}
x \\
y
\end{array}\right] \in W K_{\rho}(V)
$$

instead of

$$
\left[\begin{array}{l}
x \\
y
\end{array}\right]
$$

in order to specify the complementarity relation $\rho$ for the precise bonding between the corresponding symbols from the alphabet $V$ in the upper strand and lower strand of the molecule. Then for sticker system, languages are generated from DNA molecule sequences by initially starting from axioms $A$. The prolongation then takes place from the left to the right by using pairs $(u, v)$ of dominoes in $D$ according to the sticker operation $\mu$. Furthermore, elements of $W K_{\rho}(V)$ are called well-started doublestranded sequences if there is at least a position

$$
\left[\begin{array}{l}
a \\
b
\end{array}\right]_{\rho}^{*}
$$

with $a \neq \lambda$ and $b \neq \lambda$. The prolongation will finally stop when no blank symbol exists in the strings generated by the sticker system. Hence a complete double-stranded sequence in $W K_{\rho}(V)$ will be obtained ${ }^{12}$. Furthermore, the set of incomplete molecules are denoted by

$$
W_{\rho}(V)=L_{\rho}(V) \cup R_{\rho}(V) \cup L R_{\rho}(V),
$$

where

$$
\begin{aligned}
L_{\rho}(V) & =\left(\left(\begin{array}{c}
\lambda \\
V^{*}
\end{array}\right) \cup\left(\begin{array}{c}
V^{*} \\
\lambda
\end{array}\right)\right)\left[\begin{array}{l}
V \\
V
\end{array}\right]_{\rho}^{*}, \\
R_{\rho}(V) & =\left[\begin{array}{c}
V \\
V
\end{array}\right]_{\rho}^{*}\left(\left(\begin{array}{c}
\lambda \\
V^{*}
\end{array}\right) \cup\left(\begin{array}{c}
V^{*} \\
\lambda
\end{array}\right)\right), \\
L R_{\rho}(V) & =L_{\rho}(V) \cup R_{\rho}(V) .
\end{aligned}
$$

For two symbols $x, y \in L R_{\rho}(V)$, we have $x \Rightarrow y$ if and only if $y=\mu(u, \mu(x, v))$ for some $(u, v) \in D$, where $\mu(a, b)$ is the sticking operation of dominoes $a$ and $b$. Since the prolongation to the right is independent with the one to the left, thus $\mu(u, \mu(x, v))=$ $\mu(\mu(u, x), v)$. When $x_{1} \in A$ is used as a starting symbol and $x_{k}$ is in $W K_{\rho}(V)$, a sequence $x_{1} \Rightarrow x_{2} \Rightarrow$ $\cdots \Rightarrow x_{k}$ is obtained. This sequence is called computation in $\gamma$ with length $k-1$. We can also represent the sequence $x_{1} \Rightarrow x_{2} \Rightarrow \cdots \Rightarrow x_{k}$ as $x_{1} \Rightarrow^{*} x_{k}$. If there is no sticky end or no blank symbol present in the last symbol then the computation $x_{1} \Rightarrow^{*} x_{k}$ where $x_{k}$ is in $W K_{\rho}(V)$ is considered complete. A complete computation will produce a complete string $w$ such that $w \in W K_{\rho}(V)$. Hence the language of such strings is the language generated by $\gamma$, called the sticker language. For a complete computation $x_{1} \Rightarrow x_{2} \Rightarrow \cdots \Rightarrow x_{k}$ where $x_{1} \in A$ and $x_{k} \in W K_{\rho}(V)$, it is said to be of delay $d$ if $d \geqslant d\left(x_{i}\right)$, for $1 \leqslant i \leqslant$ $k$. Then for a sticker system $\gamma$, it is said to be of bounded delay if there is $d \geqslant 1$ such that $L_{d}(\gamma)=$ $L_{u}(\gamma)$ for $L_{d}(\gamma)$ the language of strings generated by $\gamma$ at the end of computations of delay at most $d$, and $L_{u}(\gamma)$ the unrestricted case. Furthermore, there are several restrictions of sticker system ${ }^{12}$, such as one-sided, regular, simple, simple one-sided and simple regular sticker system. The generative power of these restrictions has been investigated 
by Paun and Rozenberg ${ }^{16}$, and the results of the characterizations for families of regular, linear and recursively enumerable languages have been obtained. Besides investigating the generative power of sticker systems, some other restrictions to sticker systems have been studied ${ }^{17-19}$. These papers have shown that the generative power of a sticker system increases when some algebraic structures, probabilities and some weights are incorporated into the sticker system. New variants of weighted sticker systems have been introduced by Gan et al ${ }^{19}$, namely, weighted one-sided, weighted regular, weighted simple, weighted simple one-sided and weighted simple regular sticker systems. The finite pairs of axiom $L R_{\rho}(V)$ and dominoes and $W_{\rho}(V) \times W_{\rho}(V)$ of these new variants are associated with the weights from their chosen weighting spaces, such as $(\mathbb{Z},+)$, $\left(\mathbb{Z}^{k}, \oplus\right),\left(\mathbb{Z}^{k}, \otimes\right),\left(\mathbb{Q}^{+}, \times\right),(\mathbb{M},+)$. For the strand $z$ produced by strands $x$ and $y$ of $W_{\rho}(V)$, the weight for $\omega(z)$ is calculated from the weights $\omega(x)$ and $\omega(y)$ according to the operation $\odot$ and is defined as $\omega(z)=\omega(x) \odot \omega(y)$. Thus, several types of threshold languages generated by weighted sticker systems have been considered with different weighting spaces and cut-points. Furthermore, the threshold languages generated by weighted sticker systems and their variants have a higher generative power when compared to their respective usual sticker systems ${ }^{19}$.

A Chomsky grammar is written in the form $G=$ $(N, T, S, P)$, where $N$ is the nonterminal character, $T$ is the terminal character, $S$ is the axiom, and $P$ is the set of production rules, written in the form $u \rightarrow v$. The language generated by $G$ is denoted by $L(G)$.

The families of recursively enumerable, contextsensitive, context-free, linear, regular and finite languages are denoted by RE, CS, CF, LIN, REG and FIN, respectively. For these language families, the next strict inclusions, named Chomsky hierarchy, hold $^{13}$ :

\section{Theorem 1}

\section{FIN $\subset$ REG $\subset$ LIN $\subset \mathrm{CF} \subset \mathrm{CS} \subset \mathrm{RE}$.}

Next, we briefly cite some basic definitions and results of iterative splicing systems.

Definition 1 [Ref. 12] A sticker system is a construct of 4-tuple

$$
\gamma=(V, \rho, A, D),
$$

where $V$ is an alphabet, $\rho$ is the symmetric relation in $V, A$ is a finite subset of axioms $L R_{\rho}(V)$ and $D$ is a finite set of pairs $W_{\rho}(V) \times W_{\rho}(V)$. The language generated by the sticker system $\gamma$ is defined as

$$
L(\gamma)=\left\{w \in\left[\begin{array}{l}
V \\
V
\end{array}\right]^{*} \mid x \Rightarrow^{*} w, x \in A\right\} .
$$

There are five variants of families of sticker languages $^{12}$, namely, one-sided, regular, simple, simple one-sided and simple regular sticker languages as denoted by OSL, RSL, SSL, SOSL, and SRSL, respectively. The most general form of sticker systems is also known as the universal sticker system. The language generated by the universal sticker system is named as universal sticker language and will be denoted by USL. In 2013, another new variant of sticker systems, namely, weighted sticker systems ${ }^{19}$, have been introduced where some weights are considered in the classical model of the sticker system. Next, we will give the formal definition of weighted sticker system.

Definition 2 [Ref. 19] A weighted sticker system is a 7-tuple

$$
\gamma=\left(V, \rho, A_{\omega}, D_{\omega}, \omega, M, \odot\right),
$$

where $V$ is an alphabet, $\rho$ is the symmetric relation such that $\rho \subseteq V \times V, A_{\omega}$ is a finite set of axiom such that $A \subseteq L R_{\rho}(V) \times M, D_{\omega}$ is a finite subset of $\left(W_{\omega}(V) \times W_{\omega}(V)\right) \times M, \omega$ is a weighting function such that $\omega:\left(L R_{\omega}(V) \cup\left(W_{\omega}(V) \times W_{\omega}(V)\right)\right) \rightarrow M$, $M$ is a weighting space and $\odot$ is the operation over the weights $\omega(x), x \in L R_{\omega}(V) \cup\left(W_{\omega}(V) \times W_{\omega}(V)\right)$.

For the weighting space $M$, different sets of algebraic structures such as rational numbers, integers, real numbers, Cartesian products of the sets of numbers, or sets of matrices with integer entries can be considered. Then the operations over weights are defined with respect to the chosen weighting space.

Next, weighted sticker operations and the language generated by a weighted sticker system will be defined.

Definition 3 [Ref. 19] Given $(x, \omega(x)),(y, \omega(y))$ $\in A_{\omega}$ and $(u, \omega(u)),(v, \omega(v)) \in D_{\omega}$,

$$
[x, \omega(x)] \Rightarrow^{*}[y, \omega(y)]
$$

if and only if

(i) $[y, \omega(y)]=\mu([u, \omega(u)], \mu([x, \omega(x)],[v, \omega(v)]))$ and $\omega(y)=\omega(x) \odot \omega(v)$, and

(ii) $[y, \omega(y)]=\mu(\mu([x, \omega(x)],[u, \omega(u)]),[v, \omega(v)])$ and $\omega(y)=\omega(x) \odot \omega(u)$. 
The language generated by the weighted sticker system is defined as

$$
\begin{aligned}
\omega \mathrm{SL}=\left\{y \in W K_{\rho}(V) \mid[x, \omega(x)]\right. \\
\\
\left.\Rightarrow^{*}[y, \omega(y)] \text { for }[x, \omega(x)] \in A_{\omega}\right\} .
\end{aligned}
$$

Here, a weighted sticker system with different weights may generate the same strings. To eliminate this ambiguity, a second operation over the weights of strings has to be considered. Hence the selections on the successful subset of the language generated by a weighted sticker system with respect to some cut-points are processed to obtain the threshold languages of the weighted sticker system. We consider three types of threshold languages as stated in the next definition.

Definition 4 [Ref. 19] Let $\omega \mathrm{SL}(\gamma)$ be the language generated by a weighted sticker system $\gamma=\left(V, \rho, A_{\omega}, D_{\omega}, \omega, M, \odot\right)$. A threshold weighted sticker system language with respect to a threshold (cut-point) $\tau \in M$ is a subset of $\omega \operatorname{SL}(\gamma)$ defined by $\omega \operatorname{SL}(\gamma, \star \tau)=\left\{y \in W K_{\rho}(V) \mid[x, \omega(x)] \Rightarrow^{*}\right.$ $[y, \omega(y)]$ for $[x, \omega(x)] \in A$ and $\left.\omega(y)^{\star} \tau\right\}$, where $\star \in\{=,\langle\rangle$,$\} is called the mode of \omega \operatorname{SL}(\gamma, \star \tau)$.

Furthermore, when we consider some weights to sticker systems, we have new variants of sticker systems. The threshold languages generated by these new variants of sticker system such as weighted onesided, weighted regular, weighted simple, weighted simple one-sided, weighted simple regular, and weighted universal sticker systems will be denoted by $\omega$ OSL, $\omega$ RSL, $\omega S S L, \omega S O S L, \omega S R S L$, and $\omega$ USL, respectively. The definitions of weighted onesided, regular, simple, simple one-sided, and simple regular sticker systems are given below following Ref. 19.

Definition 5 A weighted sticker system

$$
\gamma=\left(V, \rho, A_{\omega}, D_{\omega}, \omega, M, \odot\right)
$$

is said to be one-sided if for each pair $((u, \omega(u)),(v, \omega(v))) \in D_{\omega}$ either $(u, \omega(u)) \Rightarrow$ $(\lambda, e)$ or $(v, \omega(v)) \Rightarrow(\lambda, e)$, where $e$ is the identity of the weighting space.

Definition 6 A weighted sticker system

$$
\gamma=\left(V, \rho, A_{\omega}, D_{\omega}, \omega, M, \odot\right)
$$

is said to be regular if for each pair $((u, \omega(u)),(v, \omega(v))) \in D_{\omega}$ we have $(u, \omega(u)) \Rightarrow$ $(\lambda, e)$, where $e$ is the identity of the weighting space.
Definition 7 A weighted sticker system

$$
\gamma=\left(V, \rho, A_{\omega}, D_{\omega}, \omega, M, \odot\right)
$$

is said to be simple if for all pairs $((u, \omega(u)),(v, \omega(v))) \in D_{\omega}$ either

$$
(u, \omega(u)),(v, \omega(v)) \in\left(\begin{array}{c}
\lambda \\
V^{*}
\end{array}\right) \times M
$$

or

$$
(u, \omega(u)),(v, \omega(v)) \in\left(\begin{array}{c}
V^{*} \\
\lambda
\end{array}\right) \times M .
$$

Definition 8 A weighted sticker system

$$
\gamma=\left(V, \rho, A_{\omega}, D_{\omega}, \omega, M, \odot\right)
$$

is said to be simple one-sided if for all pairs $((u, \omega(u)),(v, \omega(v))) \in D_{\omega}$ either

$$
(u, \omega(u)),(v, \omega(v)) \in\left(\begin{array}{c}
\lambda \\
V^{*}
\end{array}\right) \times M
$$

or

$$
(u, \omega(u)),(v, \omega(v)) \in\left(\begin{array}{c}
V^{*} \\
\lambda
\end{array}\right) \times M
$$

and for each pair $((u, \omega(u)),(v, \omega(v))) \in D^{\prime}$, either $(u, \omega(u)) \Rightarrow(\lambda, e)$ or $(v, \omega(v)) \Rightarrow(\lambda, e)$ with $e$ the identity of the weighting space.

Definition 9 A weighted sticker system $\gamma=\left(V, \rho, A_{\omega}, D_{\omega}, \omega, M, \odot\right)$ is said to be simple regular if for all pairs $((u, \omega(u)),(v, \omega(v))) \in D_{\omega}$, either

$$
(u, \omega(u)),(v, \omega(v)) \in\left(\begin{array}{c}
\lambda \\
V^{*}
\end{array}\right) \times M
$$

or

$$
(u, \omega(u)),(v, \omega(v)) \in\left(\begin{array}{c}
V^{*} \\
\lambda
\end{array}\right) \times M
$$

and for each pair $((u, \omega(u)),(v, \omega(v))) \in$ $D^{\prime},(u, \omega(u)) \Rightarrow(\lambda, e)$ with $e$ the identity of the weighting space.

\section{MAIN RESULTS}

In this section, we investigate the generative power of the variants of sticker systems with the presence of weights. The results for the generative power of the variants of weighted sticker systems are given in the following.

Proposition 1 For $X \in\{\mathrm{U}, \mathrm{O}, \mathrm{R}, \mathrm{S}, \mathrm{SO}, \mathrm{SR}\}$,

$$
\omega X \operatorname{SL}(b) \subseteq \omega X \operatorname{SL}(u),
$$

where $b$ is the bounded delay and $u$ is the unrestricted case. 
Proof: For the bounded delay, there is some $d \geqslant 1$ such that $L_{d}(\gamma)=L_{u}(\gamma)$ for $L_{d}(\gamma)$ the string generated by $\gamma$ in bounded delay and $L_{u}(\gamma)$ is the string generated by $\gamma$ at the end of the unrestricted complete computations. Since the dominoes of sticker systems for bounded delay cases and unrestricted cases are the same, we can easily obtain the same weights for both cases when considered in some weighting spaces. Hence $L_{\omega d}(\gamma)=L_{\omega u}(\gamma)$. Hence $\omega X \operatorname{SL}(b) \subseteq \omega X \operatorname{SL}(u)$.

Since the weighted cases for sticker systems also do not have any erasing operation, we can clearly obtain the following result:

Proposition 2 For each $X \in\{\mathrm{U}, \mathrm{O}, \mathrm{R}, \mathrm{S}, \mathrm{SO}, \mathrm{SR}\}$ and $\alpha \in\{d, u\}$,

$$
\omega X \operatorname{SL}(\alpha) \subseteq \mathrm{CS} .
$$

Now the generative power for variants of weighted sticker systems will be investigated: the relations between variants of weighted sticker systems, and the relations of the variants of weighted sticker systems in the Chomsky hierarchy. Some results on the generative power of variants of weighted sticker systems are given in the following theorems and corollary.

\section{Theorem $2 \omega \mathrm{OSL} \subseteq \omega \mathrm{REG}$}

Proof: Consider a weighted one-sided sticker system

$$
\gamma=\left(V, \rho, A_{\omega}, D_{\omega}, \omega, M, \odot\right) .
$$

We construct a weighted context-free grammar $G=$ $(N, T, S, P, M)$, such that

$$
\begin{gathered}
N=\left\{\left[\left(\begin{array}{l}
u \\
\lambda
\end{array}\right)\right]_{1},\left[\left(\begin{array}{l}
u \\
\lambda
\end{array}\right)\right]_{r},\left[\left(\begin{array}{l}
\lambda \\
u
\end{array}\right)\right]_{1},\left[\left(\begin{array}{l}
\lambda \\
u
\end{array}\right)\right]_{r} \mid\right. \\
\left.u \in V^{*}, \quad 0 \leqslant|u| \leqslant d\right\} \cup\{S\}, \\
T=\left(\begin{array}{l}
V \\
V
\end{array}\right)_{\rho} .
\end{gathered}
$$

Then by considering the weighted sticker system and weighted grammar where the weights for the production rules in weighted grammar such that

$$
S \stackrel{\omega_{1}^{\prime}}{\longrightarrow}\left[\left(\begin{array}{l}
u_{1} \\
u_{2}
\end{array}\right)\right]_{1}\left(\begin{array}{l}
x_{1} \\
x_{2}
\end{array}\right)\left[\begin{array}{l}
v_{1} \\
v_{2}
\end{array}\right]_{r}
$$

for

$$
\begin{array}{r}
\left(\left(\begin{array}{l}
u_{1} \\
u_{2}
\end{array}\right)\left(\begin{array}{l}
x_{1} \\
x_{2}
\end{array}\right)\left(\begin{array}{l}
v_{1} \\
v_{2}
\end{array}\right), \omega\left(\left(\begin{array}{l}
u_{1} \\
u_{2}
\end{array}\right)\left(\begin{array}{l}
x_{1} \\
x_{2}
\end{array}\right)\left(\begin{array}{l}
v_{1} \\
v_{2}
\end{array}\right)\right)\right) \\
\in A_{\omega},
\end{array}
$$

where

$$
\omega_{1}^{\prime}=\omega\left(\left(\begin{array}{l}
u_{1} \\
u_{2}
\end{array}\right)\left(\begin{array}{l}
x_{1} \\
x_{2}
\end{array}\right)\left(\begin{array}{l}
v_{1} \\
v_{2}
\end{array}\right)\right)
$$

with

$$
\left(\begin{array}{l}
u_{1} \\
u_{2}
\end{array}\right),\left(\begin{array}{c}
v_{1} \\
v_{2}
\end{array}\right) \in\left(\begin{array}{c}
\lambda \\
V^{*}
\end{array}\right) \cup\left(\begin{array}{c}
V^{*} \\
\lambda
\end{array}\right)
$$

and

$$
\left(\begin{array}{l}
x_{1} \\
x_{2}
\end{array}\right) \in W K_{\rho}(V)
$$

(ii)

$$
\left[\left(\begin{array}{l}
u_{1} \\
u_{2}
\end{array}\right)\right]_{1} \stackrel{\omega_{2}^{\prime}}{\longrightarrow}\left[\left(\begin{array}{l}
u_{1}^{\prime} \\
u_{2}^{\prime}
\end{array}\right)\right]_{1}\left(\begin{array}{l}
w_{1} \\
w_{2}
\end{array}\right)
$$

for

$$
\begin{gathered}
\left(\begin{array}{l}
u_{1} \\
u_{2}
\end{array}\right),\left(\begin{array}{l}
u_{1}^{\prime} \\
u_{2}^{\prime}
\end{array}\right) \in\left(\begin{array}{c}
\lambda \\
V^{*}
\end{array}\right) \cup\left(\begin{array}{c}
V^{*} \\
\lambda
\end{array}\right), \\
\left(\begin{array}{l}
w_{1} \\
w_{2}
\end{array}\right) \in W K_{\rho}(V),
\end{gathered}
$$

and there is a pair in $D_{\omega}$ of the form

$$
\left(\left(\left(\begin{array}{l}
z_{1} \\
z_{2}
\end{array}\right),\left(\begin{array}{l}
\lambda \\
\lambda
\end{array}\right)\right), \omega\left(\left(\begin{array}{l}
z_{1} \\
z_{2}
\end{array}\right)\left(\begin{array}{l}
\lambda \\
\lambda
\end{array}\right)\right)\right)
$$

and

$$
\left[\left(\begin{array}{l}
u_{1} \\
u_{2}
\end{array}\right)\right]_{r} \stackrel{\omega_{3}^{\prime}}{\longrightarrow}\left(\begin{array}{l}
w_{1} \\
w_{2}
\end{array}\right)\left[\left(\begin{array}{l}
u_{1}^{\prime} \\
u_{2}^{\prime}
\end{array}\right)\right]_{r},
$$

where

$$
\begin{gathered}
\left(\begin{array}{l}
u_{1} \\
u_{2}
\end{array}\right),\left(\begin{array}{l}
u_{1}^{\prime} \\
u_{2}^{\prime}
\end{array}\right) \in\left(\begin{array}{c}
\lambda \\
V^{*}
\end{array}\right) \cup\left(\begin{array}{c}
V^{*} \\
\lambda
\end{array}\right), \\
\left(\begin{array}{l}
w_{1} \\
w_{2}
\end{array}\right) \in W K_{\rho}(V),
\end{gathered}
$$

and there is a pair in $D_{\omega}$ of the form

$$
\left(\left(\begin{array}{l}
\lambda \\
\lambda
\end{array}\right)\left(\begin{array}{l}
z_{1} \\
z_{2}
\end{array}\right)\right)
$$

and

$$
\begin{gathered}
\omega_{3}^{\prime}=\omega\left(\left(\begin{array}{l}
\lambda \\
\lambda
\end{array}\right)\left(\begin{array}{l}
z_{1} \\
z_{2}
\end{array}\right)\right), \\
{\left[\begin{array}{l}
\lambda \\
\lambda
\end{array}\right]_{1} \stackrel{\omega_{4}^{\prime}}{\longrightarrow} \lambda,}
\end{gathered}
$$

where $\omega_{4}^{\prime}=E$

$$
\left[\begin{array}{l}
\lambda \\
\lambda
\end{array}\right]_{r} \stackrel{\omega_{5}^{\prime}}{\longrightarrow} \lambda
$$


where $\omega_{5}^{\prime}=E$ and $E \in\left\{(\mathbb{Z},+),\left(\mathbb{Z}^{k}, \oplus\right),\left(\mathbb{Z}^{k}, \otimes\right)\right.$, $\left.\left(\mathbb{Q}^{+}, \times\right),(\mathbb{M},+)\right\}$ is the identity element for each of the selected weighting spaces.

So one-sided pairs can only build the sequences by prolonging sequences to the left using rule (ii) or prolonging sequences to the right using rule (iii) independently. So with the similar argument to the proof of Theorem 4 in Ref. 16, one concludes that $\omega \mathrm{OSL} \subseteq \omega$ REG.

Theorem $3 \omega \mathbf{R E G} \subseteq \omega \mathrm{RSL}(b)$

Proof: By using the same arguments as in the proof of Theorem 7 in Ref. 16 and by considering a weighted finite automata $M=$ $\left(\mathbb{Q}, \mathbb{Z}, s_{0}, F, \delta, \omega, M, \odot\right)$ and regular sticker system $\gamma=\left(V, \rho, A_{\omega}, D_{\omega}, \omega^{\prime}, M, \odot\right)$, where

$$
\begin{aligned}
& \rho=\{(a, a) \mid a \in V\}, \\
& A_{\omega}=\left\{\left(\begin{array}{l}
x \\
x
\end{array}\right), \omega^{\prime}\left(\begin{array}{l}
x \\
x
\end{array}\right) \mid x, \omega(x) \in L_{\omega}(M),\right. \\
& |x| \leqslant n+2\} \cup\left\{\left(\left(\begin{array}{l}
x \\
x
\end{array}\right)\left(\begin{array}{l}
u \\
\lambda
\end{array}\right)\right),\right. \\
& \omega^{\prime}\left(\left(\begin{array}{l}
x \\
x
\end{array}\right)\left(\begin{array}{l}
u \\
\lambda
\end{array}\right)\right)|| x u \mid=n+2, \\
& |x| \geqslant 1,|u|=i \text { for } 1 \leqslant i \leqslant n+1 \\
& \left.: s_{0} x u \stackrel{\omega_{1}}{\rightarrow} s_{i-1}\right\} \text {, } \\
& \text { where } \omega^{\prime}\left(\left(\begin{array}{l}
x \\
x
\end{array}\right)\left(\begin{array}{l}
u \\
\lambda
\end{array}\right)\right)=\omega_{1} \text {, } \\
& D_{\omega}=\left\{\left(\left(\begin{array}{l}
\lambda \\
\lambda
\end{array}\right), \omega^{\prime}\left(\left(\begin{array}{l}
\lambda \\
\lambda
\end{array}\right)\right)\right),\left(\left(\begin{array}{l}
\lambda \\
v
\end{array}\right)\left(\begin{array}{l}
x \\
x
\end{array}\right)\left(\begin{array}{l}
u \\
\lambda
\end{array}\right),\right.\right. \\
& \left.\omega^{\prime}\left(\left(\begin{array}{l}
\lambda \\
v
\end{array}\right)\left(\begin{array}{l}
x \\
x
\end{array}\right)\left(\begin{array}{l}
u \\
\lambda
\end{array}\right)\right)\right)|1 \leqslant| v \mid \leqslant n+1, \\
& |x u|=n+2,|x| \geqslant 1,|u|=i \text { for } \\
& \left.1 \leqslant i \leqslant n+1: s_{j} x u{\stackrel{\omega_{2}}{\rightarrow}}^{*} s_{i-1}, j=|v|-1\right\} \\
& \cup\left\{\left(\left(\begin{array}{l}
\lambda \\
\lambda
\end{array}\right), \omega^{\prime}\left(\left(\begin{array}{l}
\lambda \\
\lambda
\end{array}\right)\right)\right),\left(\left(\begin{array}{l}
\lambda \\
v
\end{array}\right)\left(\begin{array}{l}
x \\
x
\end{array}\right),\right.\right. \\
& \left.\omega^{\prime}\left(\left(\begin{array}{l}
\lambda \\
v
\end{array}\right)\left(\begin{array}{l}
x \\
x
\end{array}\right)\right)\right)|1 \leqslant| v \mid \leqslant n+1 \text {, } \\
& 1 \leqslant|x| \leqslant n \text {, and } s_{j} x \stackrel{\omega}{\rightarrow}^{*} s_{\mathrm{f}}, s_{\mathrm{f}} \in F \\
& \text { for } \omega^{\prime}\left(\omega^{\prime}\left(\left(\begin{array}{l}
\lambda \\
v
\end{array}\right)\left(\begin{array}{l}
x \\
x
\end{array}\right)\left(\begin{array}{l}
u \\
\lambda
\end{array}\right)\right)\right)=\omega_{2} \text {, } \\
& \left.\omega^{\prime}\left[\begin{array}{l}
\lambda \\
v
\end{array}\right]=\omega_{3} \text { and } \omega^{\prime}\left[\begin{array}{l}
\lambda \\
\lambda
\end{array}\right]=E\right\} \text {, }
\end{aligned}
$$

where $E \in\left\{(\mathbb{Z},+),\left(\mathbb{Z}^{k}, \oplus\right),\left(\mathbb{Z}^{k}, \otimes\right),\left(\mathbb{Q}^{+}, \times\right),(\mathbb{M},+)\right\}$ is the identity element for each of the selected weighting spaces. Hence we conclude that $\omega$ REG $\subseteq$ $\omega \operatorname{RSL}(b)$.

From Proposition 1, Theorem 2 and Theorem 3, the following theorem is obtained.

Theorem $4 \omega \mathrm{OSL}=\omega \mathrm{RSL}$

Now we investigate the generative power of the universal weighted sticker system, as shown in Theorem 5.

Theorem $5 \omega \mathrm{LIN} \subseteq \omega \mathrm{USL}(b)$

Proof: Consider a weighted linear grammar $G=$ $(N, T, S, P, \omega, M)$. There is an equivalent grammar $G^{\prime}=\left(N^{\prime}, T, S, P^{\prime}, M^{\prime}\right)$ with $P^{\prime}$ containing only rules of the forms $X \stackrel{\omega}{\rightarrow} a Y, X \stackrel{\omega}{\rightarrow} Y a, X \stackrel{\omega}{\rightarrow} a$ for $X, Y \in N^{\prime}, a \in T$ (for instance, a rule $X \rightarrow$ $a_{1} a_{2} \ldots a_{n} Y b_{m} b_{m-1} \ldots b_{1}$ in $P$ can be replaced by the rules $X \rightarrow a_{1} X_{1}, X_{1} \rightarrow a_{2} X_{2}, \ldots, X_{n-1} \rightarrow a_{n} Y_{1}, Y_{1} \rightarrow$ $Y_{2} b_{1}, Y_{2} \rightarrow Y_{3} b_{2}, \ldots, Y_{m-1} \rightarrow Y_{m} b_{m-1}, Y_{m} \rightarrow b_{m}$ ). Then construct a weighted universal sticker system such that $\gamma=\left(T, \rho, A_{\omega}, D_{\omega}, \omega^{\prime}, M, \odot\right)$ where

$$
\begin{gathered}
\rho=\{(a, a) \mid a \in T\}, \\
A_{\omega}=\left\{\left(\begin{array}{l}
x \\
x
\end{array}\right), \omega^{\prime}\left(\begin{array}{l}
x \\
x
\end{array}\right) \mid x, \omega(x) \in L_{\omega}(M),\right. \\
|x| \leqslant 3 n+1\} \cup\left\{\left(\left(\begin{array}{l}
u \\
\lambda
\end{array}\right)\left(\begin{array}{l}
x \\
x
\end{array}\right)\right),\right. \\
\omega^{\prime}\left(\left(\begin{array}{l}
u \\
\lambda
\end{array}\right)\left(\begin{array}{l}
x \\
x
\end{array}\right)\right)|| x u|=3 n+1,| x \mid \geqslant 1, \\
\left.|u|=i \text { for } 1 \leqslant i \leqslant n: X_{i} \rightarrow^{*} x u\right\} \\
\cup\left\{\left(\left(\begin{array}{l}
x \\
x
\end{array}\right)\left(\begin{array}{l}
u \\
\lambda
\end{array}\right)\right), \omega^{\prime}\left(\left(\begin{array}{l}
x \\
x
\end{array}\right)\left(\begin{array}{l}
u \\
\lambda
\end{array}\right)\right) \mid\right. \\
|x u|=3 n+1,|x| \geqslant 1,|u|=i \\
\text { for } \left.1 \leqslant i \leqslant n: X_{i} \rightarrow^{*} x u\right\}
\end{gathered}
$$

and $D_{w}$ contains groups of brick pairs (1) to (6) in Ref. 16 (Theorem 8 pp.197) with the weights defined as follows:

$$
\omega^{\prime}\left(\begin{array}{l}
x \\
x
\end{array}\right)=\omega_{1}
$$

such that $\omega_{1}=\omega\left(S \Rightarrow^{*} x\right)$ (for $S \stackrel{r_{1}}{\Rightarrow} x_{1} \stackrel{r_{2}}{\Rightarrow} x_{2} \Rightarrow \cdots \Rightarrow$ $\left.r_{m} x, \omega\left(S \Rightarrow^{*} x\right)=\omega\left(r_{1}\right)+\omega\left(r_{2}\right)+\cdots+\omega\left(r_{n}\right)\right)$;

$$
\omega^{\prime}\left(\left(\begin{array}{l}
u \\
\lambda
\end{array}\right)\left(\begin{array}{l}
x \\
x
\end{array}\right)\right)=\omega_{2}
$$

such that $\omega_{2}=\omega\left(X_{i} \Rightarrow^{*} u x\right)$ for $X_{i} \Rightarrow^{*} u x$;

(iii)

$$
\omega^{\prime}\left(\left(\begin{array}{l}
x \\
x
\end{array}\right)\left(\begin{array}{l}
u \\
\lambda
\end{array}\right)\right)=\omega_{3}
$$


such that $\omega_{3}=\omega\left(X_{i} \Rightarrow^{*} x u\right)$ for $X_{i} \Rightarrow^{*} x u$;

$$
\omega^{\prime}\left(\left(\begin{array}{l}
u \\
\lambda
\end{array}\right)\left(\begin{array}{l}
x \\
x
\end{array}\right)\left(\begin{array}{l}
\lambda \\
v
\end{array}\right),\left(\begin{array}{l}
z \\
z
\end{array}\right)\right)=\omega_{4}
$$

such that $\omega_{4}=\omega\left(X_{|u|} \Rightarrow^{*} x u X_{|v|} z\right)$ for $X_{|u|} \Rightarrow^{*}$ $x u X_{|v|} z$;

$$
\omega^{\prime}\left(\left(\begin{array}{l}
x \\
x
\end{array}\right)\left(\begin{array}{l}
\lambda \\
v
\end{array}\right),\left(\begin{array}{l}
z \\
z
\end{array}\right)\left(\begin{array}{l}
u \\
\lambda
\end{array}\right)\right)=\omega_{5}
$$

such that $\omega_{5}=\omega\left(X_{|u|} \Rightarrow^{*} x X_{|v|} z u\right)$ for $X_{|u|} \Rightarrow^{*}$ $x X_{|v|} z u$;

$$
\omega^{\prime}\left(\left(\begin{array}{l}
x \\
x
\end{array}\right)\left(\begin{array}{l}
\lambda \\
v
\end{array}\right),\left(\begin{array}{l}
z \\
z
\end{array}\right)\right)=\omega_{6}
$$

such that $\omega_{6}=\omega\left(S \Rightarrow^{*} x X_{|v|} z\right)$ for $X_{|u|} \Rightarrow^{*} x X_{|v|} z$;

$$
\omega^{\prime}\left(\left(\begin{array}{l}
z \\
z
\end{array}\right),\left(\begin{array}{l}
\lambda \\
v
\end{array}\right)\left(\begin{array}{l}
x \\
x
\end{array}\right)\left(\begin{array}{l}
u \\
\lambda
\end{array}\right)\right)=\omega_{7}
$$

such that $\omega_{7}=\omega\left(X_{|u|} \Rightarrow^{*} z X_{|v|} x u\right)$ for $X_{|u|} \Rightarrow^{*}$ $z X_{|v|} x u$;

$$
\omega^{\prime}\left(\left(\begin{array}{l}
u \\
\lambda
\end{array}\right)\left(\begin{array}{l}
z \\
z
\end{array}\right),\left(\begin{array}{l}
\lambda \\
v
\end{array}\right)\left(\begin{array}{l}
x \\
x
\end{array}\right)\right)=\omega_{8}
$$

such that $\omega_{8}=\omega\left(X_{|u|} \Rightarrow^{*} u z X_{|v|} x\right)$ for $X_{|u|} \Rightarrow^{*}$ $u z X_{|v|} x$;

$$
\omega^{\prime}\left(\left(\begin{array}{l}
z \\
z
\end{array}\right),\left(\begin{array}{l}
\lambda \\
v
\end{array}\right)\left(\begin{array}{l}
x \\
x
\end{array}\right)\right)=\omega_{9}
$$

such that $\omega_{9}=\omega\left(S \Rightarrow^{*} z X_{|v|} x\right)$ for $S \Rightarrow^{*} z X_{|v|} x$.

By using the similar arguments on the weights in (1), the weights in (ii) to (ix) are also true by calculating the weights in each production rule.

To prove the theorem, we use similar arguments to the proof of Theorem 8 in Ref. 16. By using the weighted grammar and weighted universal sticker systems above, we conclude that $\omega$ LIN $\subseteq$ $\omega \operatorname{USL}(b)$.

\section{Theorem $6 \omega \mathrm{USL}(b) \subseteq \omega \mathrm{LIN}$}

Proof: Considering a weighted universal sticker system $\gamma=\left(V, \rho, A_{\omega}, D_{\omega}, \omega^{\prime}, M, \odot\right)$ and weighted grammar $G=(N, T, S, P, \omega, M)$ where

$$
\begin{aligned}
N= & \left\{\left[\left(\begin{array}{l}
u_{1} \\
u_{2}
\end{array}\right),\left(\begin{array}{l}
v_{1} \\
v_{2}
\end{array}\right)\right] \mid\left(\begin{array}{l}
u_{1} \\
u_{2}
\end{array}\right),\left(\begin{array}{l}
v_{1} \\
v_{2}
\end{array}\right) \in\left(\begin{array}{c}
\lambda \\
V^{*}
\end{array}\right)\right. \\
& \left.\cup\left(\begin{array}{l}
V^{*} \\
\lambda
\end{array}\right),\left|u_{1}\right|,\left|u_{2}\right|,\left|v_{1}\right|,\left|v_{2}\right| \leqslant d\right\} \cup\{S\}, \\
T= & \left(\begin{array}{l}
V \\
V
\end{array}\right)_{\rho}
\end{aligned}
$$

and $P$ contains the rules similar as the rules 14 in Ref. 16 (Theorem 6, pp.194-195) where the weights are assigned to the rules as follows:

$$
S \stackrel{\omega_{1}}{\longrightarrow}\left(\begin{array}{l}
w_{1} \\
w_{2}
\end{array}\right)\left[\left(\begin{array}{l}
u_{1} \\
u_{2}
\end{array}\right),\left(\begin{array}{l}
v_{1} \\
v_{2}
\end{array}\right)\right]\left(\begin{array}{l}
z_{1} \\
z_{2}
\end{array}\right),
$$

where

$$
\omega_{1}=\omega\left(\left(\begin{array}{l}
x_{1} \\
x_{2}
\end{array}\right),\left(\begin{array}{l}
y_{1} \\
y_{2}
\end{array}\right)\right)
$$

$$
\left[\left(\begin{array}{l}
u_{1} \\
u_{2}
\end{array}\right),\left(\begin{array}{l}
v_{1} \\
v_{2}
\end{array}\right)\right] \stackrel{\omega_{2}}{\longrightarrow}\left(\begin{array}{l}
w_{1} \\
w_{2}
\end{array}\right)\left[\left(\begin{array}{l}
u_{1}^{\prime} \\
u_{2}^{\prime}
\end{array}\right),\left(\begin{array}{l}
v_{1}^{\prime} \\
v_{2}^{\prime}
\end{array}\right)\right]\left(\begin{array}{l}
z_{1} \\
z_{2}
\end{array}\right)
$$

where

$$
\omega_{2}=\omega\left(\left(\begin{array}{l}
x_{1} \\
x_{2}
\end{array}\right),\left(\begin{array}{l}
y_{1} \\
y_{2}
\end{array}\right)\right)
$$

(iii) $\left[\left(\begin{array}{l}u_{1} \\ u_{2}\end{array}\right),\left(\begin{array}{l}v_{1} \\ v_{2}\end{array}\right)\right] \stackrel{\omega_{3}}{\longrightarrow}\left(\begin{array}{l}w_{1} \\ w_{2}\end{array}\right)\left[\left(\begin{array}{l}x_{1} \\ x_{2}\end{array}\right),\left(\begin{array}{l}z_{1} \\ z_{2}\end{array}\right)\right]\left(\begin{array}{l}z_{1} \\ z_{2}\end{array}\right)$,

where

$$
\begin{gathered}
\omega_{3}=\omega\left(\left(\begin{array}{l}
w_{1}^{\prime} \\
w_{2}^{\prime}
\end{array}\right)\left(\begin{array}{l}
x_{1} \\
x_{2}
\end{array}\right)\left(\begin{array}{l}
z_{1}^{\prime} \\
z_{2}^{\prime}
\end{array}\right)\right), \\
S \stackrel{\omega_{4}}{\longrightarrow}\left(\begin{array}{l}
w_{1} \\
w_{2}
\end{array}\right),
\end{gathered}
$$

where

$$
\omega_{4}=\omega\left(\left(\begin{array}{l}
w_{1} \\
w_{2}
\end{array}\right)\right)
$$

Following similar arguments to the proof of Theorem 6 in Ref. 16, by considering the weighted grammar $G$ defined above, we can obtain $L_{d}(\gamma) \in$ $\omega$ LIN. Hence $\omega \operatorname{USL}(b) \subseteq \omega$ LIN .

Using Theorem 5 and Theorem 6 , the following corollary is obtained.

Corollary $1 \omega \mathrm{USL}(b)=\omega \mathrm{LIN}$

Acknowledgements: The second and third authors were supported by MOE through UTM Research University Grant Vote No. 13H18. The fourth author is grateful to the Ministry of Education and Research Management Centre, International Islamic University Malaysia for the financial funding through RIGS16-368-0532.

\section{REFERENCES}

1. Hassan S, Humaira Asghar M (2010) Limitation of silicon based computation and future prospects. In: Second International Conference on Communication Software and Networks, Singapore, pp 559-61.

2. Adleman L (1994) Molecular computation of solutions to combinatorial problems. Science 266, 1021-4. 
3. Lipton R (1995) Using DNA to solve NP-complete problems. Science 268, 542-5.

4. Boneh D, Dunworth C, Lipton R, Sgall J (1996) On the computational power of DNA. Discrete Appl Math 71, 79-94.

5. Kari L, Paun G, Rozenberg G, Salomaa A, Yu S (1998) DNA computing, sticker systems and universality. Acta Inform 35, 401-20.

6. Salomaa A (1969) Probabilistic and weighted grammars. Inform Contr 15, 529-44.

7. Fu KS, Li T (1969) On stochastic automata and languages. Inform Sci 1, 403-19.

8. Mizumoto M, Toyoda J, Tanaka K (1973) Examples of formal grammars with weights. Inform Process Lett 2, 74-8.

9. Mizumoto M, Toyoda J, Tanaka K (1975) Various kinds of automata with weights. $J$ Comput Syst Sci 10, 219-36.

10. Alexandrakis A, Bozapalidis S (1987) Weighted grammars and Kleene's theorem. Inform Process Lett 24, 1-4.

11. Droste M, Kuich W, Vogler H (2009) Handbook of Weighted Automata, Springer-Verlag, Berlin.

12. Păun G, Rozenberg G, Salomaa A (1998) DNA Computing: New Computing Paradigms, Springer-Verlag, Berlin.

13. Rozenberg G, Salomaa A (1997) Handbook of Formal Languages, Springer-Verlag, Berlin.

14. Dassow J, Păun G (1989) Regulated Rewriting in Formal Language Theory, Springer-Verlag, Berlin.

15. Linz P (2006) An Introduction to Formal Languages and Automata, Jones and Bartlett Publishers, USA.

16. Păun G, Rozenberg G (1998) Sticker system. Theor Comput Sci 204, 183-203.

17. Mohd Sebry NA, Hamzah NZA, Sarmin NH, Fong WH, Turaev S (2012) Sticker systems over monoids. Malays J Fund Appl Sci 8, 131-6.

18. Selvarajoo M, Fong WH, Sarmin NH, Turaev S (2013) Probabilistic sticker systems. Malays J Fund Appl Sci 9, 150-5.

19. Gan YS, Fong WH, Sarmin NH, Turaev S (2013) Some properties and variants of weighted sticker system. Int J Appl Math Stat 45, 367-75. 